\title{
El éxito del Mercosur posible
}

ALDO FERRER*

The success of the possible Mercosur. Mercosur can be observed from two different perspectives. One from an ideal integration project, whose reference is the European Union. The other, based in the profound prevailing asymmetries within the region and the progress achieved since the founding bilateral agreements of presidents Alfonsin and Sarney, in 1985. From the first perspective, Mercosur in a failure; from the second, it has achieved considerable success. The integration process is displayed in three levels: the national density prevailing in the member countries, the rules of the game of the system and the common standings vis a vis the rest of the world. The future of Mercosur depends on progress achieved in these three levels and the opening of new possibilities of national development for each member country in a regional framework.

Key-words: globalization, national density, flexibility, economic development, public policies, market forces.

JEL Classification: F02; O1

La evaluación de los logros del Mercosur da lugar a dos aproximaciones distintas. Una de ellas desde la perspectiva del Mercosur ideal; la otra, desde la del Mercosur posible.

La primera compara la situación actual del sistema regional con el objetivo del mercado común con libre movilidad de bienes y de factores de la producción y un arancel externo común (AEC) frente al resto del mundo y, a partir de allí, la marcha hacia la plena unión económica y monetaria. El patrón de referencia es la Unión Europea incluyendo sus instituciones comunitarias (Comisión, Parlamento, Tribunal de Justicia, Banco Central) y la moneda común.

Desde esta perspectiva, el Mercosur ha fracasado porque no ha logrado cumplir los requisitos de la existencia de un mercado común. La aplicación del AEC registra múltiples excepciones (perforaciones), los países no han internalizado las

* Profesor Titular Consulto. Director de la Maestría Mercosur. Universidad de Buenos Aires. E-mail: aldo_susana_ferrer@ciudad.com.ar. Submetido: fevereiro, 2006; aceito: abril, 2006 
normas comunitarias en sus ordenamientos internos y aplican normas propias de una zona de libre comercio (como las reglas de orígen). Recientemente, el acuerdo (mecanismo de adaptación competitiva, MAC) entre Argentina y Brasil, para facilitar la recuperación industrial argentina, introduce otras excepciones al funcionamiento del mercado común A su vez, el andamiaje institucional carece de capacidad para establecer políticas comunitarias y resolver los diferendos planteados. La declinación del intercambio regional a partir de 2002 y los contínuos reclamos de agentes económicos de los países miembros sobre diversos aspectos de la marcha de la integración, incluyendo los planteos de los dos socios de menor dimensión (Paraguay y Uruguay), configuran esta impresión de fracaso que prevalece actualmente en la evaluación de la marcha del Mercosur.

Desde la perspectiva del Mercosur posible, las conclusiones son totalmente distintas. La misma toma nota de dos cuestiones principales. Por una parte, la comparación de la situación actual con la existente al tiempo de la firma, hace veinte años, del acta de Foz de Iguazú, por los presidentes Alfonsín y Sarney, que constituye el punto de partida de la formación del Mercosur. Por otra, las asimetrías en las situaciones nacionales de los países miembros y su impacto sobre el proceso de integración.

Respecto de la primera cuestión, se observa el crecimiento de la participación de los intercambios intraregionales dentro del comercio exterior de los integrantes del sistema, el estrechamiento de las relaciones en todos los planos incluyendo la adopción de posiciones conjuntas frente al resto del mundo (como en el caso de ALCA y la OMC), la resolución definitiva de antiguas desinteligencias entre Argentina y Brasil (como, por ejemplo, los objetivos del desarrollo nuclear) y la construcción de una infraestructura de transportes y energética compartida. Los avances son considerables y marcan una diferencia notable con la situación existente en el punto de partida.

Sobre la segunda cuestión, debe recordarse que el Mercosur es un espacio de profundas asimetrías. Estas lo caracterizan y diferencian de los acuerdos de integración existentes en otras partes del mundo. Dos de sus países miembros, Brasil y Argentina, representan el $95 \%$ del territorio, la población, la producción y el comercio. La incorporación de Venezuela modificará parcialmente estas proporciones. A su vez, entre Brasil y Argentina han surgido asimetrías de sus estructuras industriales y escalas de plantas que condicionan la competitividad de las producciones nacionales al interior de la región y frente al resto del mundo.

Las asimetrías observables en el Mercosur no se reducen a las diferencias entre países. En cada uno de ellos, en particular, en los extensos territorios de Argentina y Brasil, existen, asimismo, desigualdades profundas en la distribución del ingreso y en el desarrollo de sus diversas regiones y sectores productivos. Este hecho aumenta la complejidad de la integración regional y no puede ser abordado, como sucedió en el caso de la Unión Europea, con programas comunitarios para atender a las zonas rezagadas de países miembros.

Dadas estas realidades, la perspectiva del Mercosur ideal es inaplicable en la evaluación de los resultados del sistema. En cambio, desde la del Mercosur posible lo alcanzado es en verdad notable y un éxito de la decisión original de poner en marcha la convergencia de nuestros países. 
Es preciso evitar reclamarle al Mercosur lo que, al menos en el futuro previsible, no puede proporcionar. A tales fines, debe recordarse que la integración se despliega en tres planos: la situación interna de los países miembros, las reglas del juego de la integración y la proyección conjunta hacia el resto del mundo. Detengámonos, brevemente, en cada uno de estos tres planos.

\section{LAS SITUACIONES NACIONALES}

El avance de la integración depende, en gran medida, de materias propias de la situación interna de los países y que solo tienen resolución dentro de cada espacio nacional. Tales, por ejemplo, las políticas sociales para elevar el nivel de vida y ampliar el mercado interno, las políticas macroeconómicas para consolidar la gobernabilidad del presupuesto, la moneda y el balance de pagos, las reglas del juego necesarias para abrir espacios de rentabilidad que fortalezcan la competitividad e impulsen la inversión y el empleo. En realidad, la mayor parte de los conflictos al interior del Mercosur obedecen a problemas internos de los países, a sus dificultades para resolver cuestiones como las mencionadas, las cuales, inevitablemente, se proyectan a la esfera regional.

Cada uno de nuestros países procesa los cambios en curso conforme a su potencial de recursos disponibles pero, esencialmente, por sus realidades internas, vale decir, la cohesión social, la distribución del poder y la calidad de los liderazgos, la fortaleza de las instituciones y la capacidad de arbitraje del sistema político para contener los conflictos en límites manejables. Estos elementos, que componen lo que podemos llamar la densidad nacional, ${ }^{1}$ son determinantes de los carriles por los cuales cada uno de nuestros países genera y procesa los reclamos de sus propias sociedades por más bienestar y menos desigualdad. De allí la diversidad de caminos que están adoptando los gobiernos de la región, aún estando en sintonía en una inclinación generalizada hacia políticas más progresistas.

El análisis comparado de las situaciones nacionales contribuye a explicar las divergencias en las políticas adoptadas por Argentina y Brasil. En este, en la actualidad, los objetivos sociales del gobierno del Presidente Lula se insertan en una política financiera rigurosamente ortodoxa, reveladora de la solidez del dominio de los grupos económicos hegemónicos, en particular del sector financiero, que prolonga su influencia sea cual fuere la fuerza política en el gobierno. Esta situación es una condición de la relativa estabilidad de largo plazo de la política y los objetivos económicos del Brasil pero, también, una restricción a la transformación necesaria. Vale decir, el logro de una tasa de crecimiento a la altura de la extraordinaria dotación de recursos materiales y humanos del país y de la necesidad de resolver la desigualdad y la pobreza prevalecientes.

En el caso argentino, el reparto del poder está menos consolidado y, por eso, tradicionalmente, los cambios políticos suelen ser acompañados de tensiones ex-

${ }^{1}$ A. Ferrer. La densidad nacional: el caso argentino. Capital Intelectual. Buenos Aires, 2004. 
tremas y cambios radicales de rumbo, que se reflejan en la inestabilidad de largo plazo del sistema. Este rasgo de la realidad argentina contribuye a explicar como el país pudo pasar del paradigma desarrollista que prevaleció hasta el golpe de estado de 1976, a la hegemonía neoliberal que culminó con las políticas de la década de 1990 y, de aquí, a las políticas del Gobierno del Presidente Kirchner, inspiradas en una visión nacional, desarrollista, progresista. No se trata de cambios superficiales sino de desplazamientos drásticos de las políticas públicas con efectos profundos en la asignación de los recursos, la estructura productiva, la inserción internacional y la distribución de la riqueza. E, inevitablemente, también en el comportamiento de la Argentina dentro del Mercosur.

\section{LAS REGLAS DEL JUEGO DE LA INTEGRACIÓN}

Respecto de este segundo plano, debe recordarse que la integración es útil en cuanto instrumento de los países para impulsar su desarrollo nacional y fortalecer su posición en el escenario global. Sus reglas del juego deben ser, por lo tanto, consistentes con tal objetivo y distribuir, en todo el espacio regional, los beneficios de la aplicación de la ciencia y la tecnología. Esto implica la formación de economías nacionales diversificadas y complejas y una división del trabajo basada en la especialización intraindustrial. La integración necesaria y posible es incompatible con la formación, al interior del Mercosur, de un modelo centro-periferia que concentre, en cualquiera de los países, las actividades de mayor densidad tecnológica y reduzca al resto a la función de proveedores de productos primarios.

De este modo, las reglas del juego de la integración deben ser compatibles con el desarrollo el Brasil, la reindustrialización de Argentina y la apertura de espacios de rentabilidad para Paraguay y Uruguay para ampliar su acceso al mercado regional y acelerar su transformación productiva.

\section{POSICIONAMIENTO FRENTE AL RESTO DEL MUNDO}

Por último, la integración se proyecta al escenario global a través de la concertación de posiciones conjuntas en los foros multilaterales como la OMC, las negociaciones con Estados Unidos, la Unión Europea y las potencias emergentes en la cuenca de Océano Pacífico y, también, en cuestiones críticas como las de seguridad y la lucha contra el narcotráfico.

En América Latina, la integración esta condicionada por las diversas estrategias de los países respecto de su inserción con el resto del mundo, particularmente con los Estados Unidos y la Unión Europea. El Tratado de Libre Comercio de Mexico con Estados Unidos y Canadá, es el caso más notable. Cuando un país establece relaciones especiales, a través de acuerdos de libre comercio, particularmente con las potencias industriales, reduce el campo de la integración posible con los otros países de la región en la esfera de las preferencias comerciales recí- 
procas y cuestiones críticas como el tratamiento de la propiedad intelectual, los movimientos de capitales y las compras públicas. En este sentido, el espacio donde se ha preservado la mayor libertad posible en el establecimiento de los cauces de la integración regional, es el Mercosur. La situación quedo expuesta en el diferendo de la Cumbre Presidencial de Mar del Plata, noviembre 2004, respecto de la formación del ALCA. Por esto mismo, en el Mercosur se expresan con mayor claridad los conflictos y convergencias que emergen de las situaciones nacionales y de la integración, en un contexto mundial globalizado.

\section{LA CONVERGENCIA DE LOS TRES PLANOS DE LA INTEGRACIÓN}

Es en este escenario de profundas asimetrías, nacionales y dentro de la región, en donde se despliegan, simultáneamente, los tres planos de la integración... No existe una secuencia cronológica entre las acciones en las tres esferas. Cuanto más se consoliden las situaciones nacionales mas fluídos serán los intercambios, cuanto más flexibles y realistas las normas mejor serán las respuestas frente a los cambios en las situaciones nacionales y, finalmente, cuanto más solidaria sea la proyección conjunta en el escenario global, más libertad de maniobra tendrán las políticas nacionales y comunitarias.

Considerar la existencia de esos tres planos en que se despliega el Mercosur es esencial para identificar el orígen de las controversias y poder resolverlas. Por ejemplo, la asimetría de las estrategias de desarrollo seguidas por Argentina y Brasil durante prolongados períodos, generó una división del trabajo en el intercambio bilateral con rasgos del modelo centro periferia, con Argentina en la posición predominante de abastecedor de bienes de menor contenido relativo de tecnología y valor agregado. La asimetría se expresa en sectores como el automotriz, bienes de capital, electrodomésticos, informática, telecomunicaciones, calzado y textiles. Una vez que Argentina resuelve cambiar el rumbo e impulsar sus reindustrialización, necesariamente debe cambiar la dinámica y el contenido de comercio bilateral. Se comprende que si las reglas del juego de Mercosur son incompatibles con la nueva situación argentina, deben adaptarse para renovar la vigencia de la integración en el nuevo escenario. Este es el sentido del MAC, recientemente acordada por Argentina y Brasil.

Lo mismo sucede con la situación de los países de menor dimensión para los cuales, también, el Mercosur es una opción válida en la medida en que contribuya a sus desarrollos nacionales.

Aclarar estas cuestiones es vital para el futuro del Mercosur. Esta adecuación de las reglas del juego de la integración para responder a las asimetrías y a los cambios en las realidades nacionales, puede entenderse, según se la observe desde la perspectiva ideal o posible, como un fracaso de la integración o como un éxito.

Si se ve como un fracaso es porque se supone que, dadas las reglas existentes, la integración consiste en dejar librado a la aparente irreversibilidad de las asimetrías y a las fuerzas del mercado, el curso de los acontecimientos. En consecuencia, cualquier corrección de las normas que restrinja o reoriente el intercam- 
bio, es visto como un retroceso y, aún el anticipo del final del Mercosur. Se trata de una visión reducida al contenido comercial de la integración, que es insuficiente para comprender y administrar el proceso.

En cambio, si la integración se concibe como una combinación virtuosa de fuerzas del mercado y políticas publicas que armonizan los intereses de los países miembros, el caso del MAC, por ejemplo, puede entenderse como un éxito del Mercosur, revelador de la lucidez de las dirigencias de ambos países para preservar el objetivo estratégico, reconociendo el cambio de las circunstancias internas de sus países miembros.

Es claro que las adaptaciones no pueden ser discrecionales y deben surgir de negociaciones entre las partes, para preservar la máxima estabilidad posible de las normas. Esto es indispensable para fortalecer la competencia en el mercado ampliado y la competitividad sistémica de las producciones nacionales de bienes transables, en el mercado regional y el resto del mundo. Pero un sistema inflexible, si entra en conflicto con objetivos irrenunciables de los países miembros, se rompe.

\section{LA AGENDA FUTURA DEL MERCOSUR}

El Mercosur sigue siendo el emprendimiento más profundo de la integración latinoamericana y conserva potencial de crecimiento, siempre y cuando se sostenga sobre el impulso al pleno desarrollo de sus países miembros.

El futuro del Mercosur depende, en gran medida, de dos condiciones básicas. Por una parte, comprensión y tolerancia entre Argentina y Brasil para procesar los cambios en sus respectivas situaciones nacionales y, por otra, acuerdos para ampliar las oportunidades que el Mercosur ofrece a Paraguay y Uruguay.

Es preciso construir el Mercosur posible, avanzando con firmeza en las amplias fronteras abiertas a la convergencia entre nuestros países. Desde esta perspectiva, surgen diversas cuestiones prioritarias. Como las siguientes:

Las instituciones. Acerca de la conducción del proceso, conviene recordar que las asimetrías de tamaño de los países miembros, en un sistema de solo cuatro integrantes y, previsiblemente, cinco, impiden establecer, al estilo de la Unión Europea, instituciones supranacionales de integración en las cuales delegar, por ejemplo, la solución de las controversias o el establecimiento de políticas comunitarias. El Mercosur requiere, como condición necesaria, el acuerdo de Argentina y Brasil, cuya convergencia durante los gobiernos de Alfonsin y Sarney, constituye la piedra fundacional del sistema. Existe un espacio importante para el fortalecimiento de las instituciones del Mercosur, incluyendo la incorporación de la normativa Mercosur dentro de los regímenes legales y administrativos de los países miembros. Pero sería ilusorio pensar que los diferendos que existen, o pueden surgir, entre Argentina y Brasil, pueden ser resueltos en la esfera comunitaria, sin un previo acuerdo entre las partes involucradas.

Las prioridades. Convendría concentrar las acciones en los puntos críticos del sistema. Es decir, aquellos con mayor potencial de derrame sobre el desarrollo de los países y la misma integración. Tal, por ejemplo, la infraestructura de 
transportes y comunicaciones y, en particular, la energética. Más allá del gas, el petróleo y la hidroelectricidad, otras fuentes de energía ofrecen inmensas posibilidades de integración, como es el caso de la energía nuclear. En este terreno, la complementación del parque nucleoeléctrico de Argentina y Brasil, con cuatro centrales en operación y dos en construcción, ofrece una inmensa posibilidad de desarrollo, con derrames en los sistemas nacionales de ciencia y tecnología. Este fue el objetivo de la creación de la AABAEN (Agencia Argentino Brasileña de Cooperación en Energía Nuclear), durante mi desempeño en la Presidencia del Directorio de la Comisión Nacional de Energía Atómica.

La programación sectorial. Los acuerdos sectoriales de integración industrial, como el de bienes de capital, constituían un instrumento clave de los acuerdos bilaterales firmados por los presidentes Alfonsín y Sarney. Tenían el objetivo de abrir espacios de rentabilidad en todos los países miembros, impulsar las inversiones y promover la especialización intraindustrial. Esta estrategia fue abandonada una vez que, bajo las presidencias de Menem y Collor, el proceso fue delegado, en las fuerzas del mercado, en un cronograma acelerado de desgravación lineal y automática del universo, arancelario. Es decir, ignorar la realidad e intentar construir el Mercosur ideal proceso que, inevitablemente, terminó en los conflictos actuales.

Reactivar la programación del desarrollo industrial conjunto a través de acuerdos en sectores claves, como bienes de capital, informática, telecomunicaciones y biotecnología, celulosa y papel, abriría espacios de rentabilidad que atraerían, en primer lugar, a empresas y recursos regionales, cuyo protagonismo es esencial para la consolidación del Mercosur. Contribuiría, también, en la resolución de los litigios planteados en diversos bienes por las asimetrías existentes en las capacidades productivas nacionales y en la composición del intercambio.

Lo mismo cabe afirmar respecto de la programación de la integración científica y tecnológica, en donde es preciso reactivar objetivos de los acuerdos bilaterales argentino brasileños, como fueron los referidos a biotecnología e informática. La cooperación en materia de defensa y equipamientos militares fabricados en la región, se ubica en el mismo contexto de la programación sectorial en áreas claves. Asimismo, la afinidad cultural entre los pueblos de la región, ofrece un inmenso espacio de posibilidades para emprendimientos conjuntos de respaldo a las múltiples expresiones de la creatividad, universalmente reconocidas, de nuestras sociedades.

Comercio. En materia comercial, la flexibilidad recomendable de las normas, para atender los cambios de circunstancias en los países miembros, debería ser acompañado por la simplificación y transparencia de las excepciones al Arancel Externo Común (AEC) y los regímenes de admisión temporaria y de zonas francas. Es preciso construir un espacio aduanero común evitando cobrar dos veces el AEC cuando un bien pasa de un país a otro como, así también, armonizar la aplicación de las normas sanitarias para evitar su utilización como método encubierto de restricción al comercio. Las normas de orígen, propias de las zonas de libre comercio e impropias de una unión aduanera, deben ser administradas con prudencia para asegurar que el comercio se refiera a bienes efectivamente de orígen regional y, al mismo tiempo, evitar que se conviertan en obstáculos al in- 
tercambio. Es preciso armonizar, también, los regímenes de incentivos a la radicación de inversiones y al fomento de las exportaciones, que distorsionan la competencia e impide la participación equitativa de los países miembros en los frutos de la ampliación del mercado.

Políticas macroeconómicas. Es improbable que pueda avanzarse en la coordinación de las políticas macroeconómicas por las diferencias de las situaciones internas de cada país. Las mismas se reflejan en las asimetrías de las paridades cambiarias, las tasas interés, el nivel de la actividad económica y la evolución de los precios. La experiencia comparada de Argentina y Brasil revela como las respectivas situaciones macroeconómicas, a través, principalmente, de los cambios en el nivel de actividad y la modificación de la paridad del peso y el real, repercuten en las corrientes de comercio bilateral y de inversiones privadas directas extranjeras.

Es improbable, al menos en plazos previsibles, que se logre armonizar el comportamiento de las paridades del peso y el real, las tasas de interés y la evolución del nivel de actividad, a través de la coordinación de las políticas fiscal, monetaria y de balance de pagos. Cabe esperar que las asimetrías en las variables macroeconómicas continúen modificando las condiciones de competitividad y las corrientes de intercambio. Serán otros instrumentos los que deban emplearse para preservar los equilibrios dentro del sistema y el reparto equitativo de los beneficios de la integración. Ente ellos, el uso prudente de cláusulas de salvaguardia, la programación conjunta de inversiones en el seno de los acuerdos sectoriales, incluyendo programas comunes de inversión pública y el crédito para proyectos compartidos. Sobre esto último está pendiente la iniciativa de crear una institución financiera de desarrollo del Mercosur o, alternativamente, programas de cooperación entre los bancos públicos y privados para el financiamiento de proyectos comunitarios y el intercambio.

La aparente inviabilidad de la coordinación de las políticas macroeconómicas no impide avanzar, dentro de ciertos límites, con la iniciativa de crear una moneda común aplicable, inicialmente, para los intercambios de bienes y servicios, incluyendo el turismo. La existencia de una moneda común para tales fines contribuiría a la formación del espíritu comunitario

Empresas locales e inversión extranjera. Una de las mas importantes contribuciones potenciales del Mercosur, al desarrollo de los países miembros, es fortalecer el papel de las empresas locales en las inversiones y el intercambio intraregional. Las filiales de las corporaciones transnacionales que operan en la región, tienen una política de su posicionamiento en el área que refleja la visión estratégica de sus respectivas matrices. Cuentan, así, con una ventaja competitiva respecto de las empresas locales, cuyo rol debe y puede ser fortalecido. A tales fines, son necesarios la armonización de los regímenes de inversión extranjera e incentivos para la proyección regional de empresas locales y la formación de empresas de capital mercosureño. Los acuerdos sectoriales de integración contribuirían a ofrecer a las firmas locales una visión regional para la toma de decisiones.

Por último, esta enumeración parcial de las áreas de la agenda de avance del Mercosur, incluye la liberalización del tránsito de ciudadanos de los países miembros, la armonización de los regímenes laborales y el reconocimiento, bajo pautas acordadas, de títulos expedidos por las instituciones académicas regionales. 


\section{EL CASO DE LAS PAPELERAS DE FRAY BENTOS}

El actual diferendo argentino uruguayo en la materia puede ser observado en el contexto de las reflexiones anteriores.

Los psicoanalistas distinguen entre los contenidos manifiestos y latentes de los problemas que analizan. Los primeros se refieren a los que están a la luz del día y son evidentes. Los segundos, a cuestiones más profundas, no aparentes, pero que contienen las explicaciones de fondo de la cuestión.

La distinción es aplicable al actual diferendo en torno de la construcción de las papeleras en la costa oriental del Río Uruguay, en Fray Bentos, frente a Gualeguaychú. El aspecto manifiesto del problema es el reclamo argentino de seguridades sobre la viabilidad ecológica de los proyectos y la defensa del medio ambiente de un espacio compartido entre los dos países. Del lado uruguayo, no se desconoce la necesidad de dar plenas garantías a la Argentina sobre la cuestión ambiental, sin limitar su derecho soberano de promover las inversiones y el desarrollo. El principal reclamo uruguayo se refiere al corte de los puentes binacionales y demanda normalizar el tráfico en una zona vital para ambos países y para el Mercosur.

El conflicto manifiesto fue demasiado lejos. Sin embargo, a esta altura, parece que ambos gobiernos están buscando la salida mediante, por una parte, un régimen de garantías efectivas, mutuamente verificadas, de que las empresas emplearán la mejor tecnología disponible para garantizar la indispensable defensa del habitat compartido y, por la otra, la normalización del tráfico en la región. Es probable que, próximamente, el diferendo sea resuelto.

Pero queda el otro aspecto de la cuestión, es decir, el contenido latente del problema. Surgen así dos interrogantes.

Primero: ¿Cómo es posible que, en un espacio compartido, proyectos de gran escala que afectan al desarrollo y al medio ambiente de toda la región puedan ser llevados hasta un avanzado nivel de ejecución sin acuerdo previo entre los países?

Segundo: ¿Por qué estos proyectos se despliegan en la forma tradicional de la inversión privada directa, con la extranjerización lisa y llana del control de las firmas, siendo que es posible concebir empresas (joint ventures) con participación decisiva de capitales propios, argentinos, uruguayos y mercosureños, con el aporte complementario necesario de las empresas extranjeras?

La respuesta es clara: esto sucede porque el Mercosur abandonó el empleo de la programación del desarrollo industrial de la región, en los sectores claves (como lo es el de celulosa y papel), que estaba previsto y puesto en práctica (en el sector de bienes de capital) en los acuerdos bilaterales argentino-brasileños, durante las presidencias de Alfonsín y Sarney. El instrumento quedó contemplado en el Tratado de Asunción pero se lo llevó el viento durante el temporal neoliberal que asoló a nuestros países.

Imaginemos un escenario distinto del desarrollo de las papeleras de Fray Bentos, en el contexto de una estrategia compartida de desarrollo industrial. Uruguay toma la iniciativa de desarrollar inversiones en el sector y lo acuerda con Argentina en un espíritu de abrir nuevas oportunidades a los socios de menor dimensión del Mercosur. Empresarios de los dos países, que no nos faltan, segura- 
mente propondrían la formación de una empresa binacional, integrada con el tejido industrial y tecnológico del Mercosur, para la realización de los proyectos, movilizando el aporte extraregional de tecnología y financiamiento que fuera necesario, pero manteniendo el control de las firmas. En tal caso, contribuiríamos a construir capitalismos nacionales en Argentina y Uruguay y, al final, un capitalismo mercosureño, con el pleno desarrollo industrial y tecnológico de los países miembros, con empleo de calidad, sin pobres y con equidad.

Las papeleras de Fray Bentos son un resabio del viejo modelo centro periferia. Vale decir, la extracción de recursos naturales de nuestros países, bajo la gestión de filiales de empresas extranjeras, destinada a la exportación al mercado mundial. Este modelo, como lo revela la experiencia, no resuelve el problema del desarrollo sustentable. Uruguay, ni la Argentina, serán prósperos si predominan este tipo de inversiones $y$, de paso, pueden enfrentar problemas como los actuales, en un diferendo inconcebible y absurdo entre argentinos y uruguayos.

Ahora hay que resolver el aspecto manifiesto del problema. Es probable que la solución esté cerca porque es claro el reclamo de nuestras sociedades y la intención de los gobiernos, más allá del despliegue de sus estrategias en esta fase confrontativa, de ponerse de acuerdo.

Pero esta historia no puede repetirse y debe dejarnos enseñanzas fecundas para el futuro. Argentinos, uruguayos, brasileños, paraguayos y sus socios en el Mercosur, para generar producción y empleo, debemos construir sistemas productivos integrados y complejos capaces de asimilar y generar conocimientos en todo el tejido económico y social. Tenemos que abrirnos al mundo, manteniendo el control de nuestro propio destino en el orden global. Debemos aprender de nuestra experiencia y de la ajena y observar que no existen países exitosos fundados sobre capitalismos vulnerables y dependientes, desigualdad y pobreza, como, prevalece en nuestra realidad actual.

Si encaramos juntos el desafío somos mucho más fuertes. Esta es la agenda pendiente del Mercosur posible. Tenemos que construir los instrumentos esenciales de la integración: uno de ellos, como hemos recordado, es el acuerdo sectorial de desarrollo compartido sobre sectores claves, para beneficio de todos y atendiendo a las asimetrías actuales. Así convertiremos problemas, como el de las papeleras de Fray Bentos, en oportunidades de desarrollo, empleo y construcción nacional de nuestros países, en el espacio solidario del Mercosur.

Lo mismo puede decirse de otros diferendos, como el planteado en electrodomésticos, calzados, textiles y otros bienes, entre Argentina y Brasil. Estas cuestiones tienen también su contenido manifiesto, al cual atiende el reciente MAC. Pero el contenido latente es que el Mercosur solo es útil a sus miembros como una herramienta efectiva de su propio desarrollo. Dadas las asimetrías existentes, de tamaño y nivel relativo de desarrollo, el mercado, librado a sus propias fuerzas, multiplica los conflictos y, en definitiva, lleva al desencuentro y la parálisis. Deben así introducirse las políticas públicas comunitarias en el Mercosur, entre las cuales, la programación industrial es uno de los principales instrumentos disponibles.

El Mercosur posible está disponible a nuestros países para construir los desarrollos nacionales en el espacio de la integración. 